\title{
Correction: NG2 antigen is involved in leukemia invasiveness and central nervous system infiltration in MLL-rearranged infant B-ALL
}

\author{
C. Prieto ${ }^{1} \cdot$ B. López-Millán ${ }^{1} \cdot$ H. Roca-Ho ${ }^{1} \cdot$ R. W. Stam ${ }^{2,3} \cdot$ D. Romero-Moya ${ }^{1} \cdot$ F. J. Rodríguez-Baena ${ }^{4}$. \\ A. Sanjuan-Pla ${ }^{7}$ V. Ayllón ${ }^{4} \cdot$ M. Ramírez ${ }^{5} \cdot$ M. Bardini ${ }^{6} \cdot$ P. De Lorenzo ${ }^{7} \cdot$ M. G. Valsecchi ${ }^{7} \cdot$ M. Stanulla ${ }^{8} \cdot$ M. Iglesias $^{9}$. \\ P. Ballerini ${ }^{10} \cdot$ Á. M. Carcaboso ${ }^{11} \cdot$ J. Mora ${ }^{11} \cdot$ F. Locatelli ${ }^{12} \cdot$ A. Bertaina ${ }^{12} \cdot$ L. Padilla $^{13}$. \\ Juan Carlos Rodríguez-Manzaneque ${ }^{4} \cdot$ C. Bueno ${ }^{1,14} \cdot P$. Menéndez ${ }^{1,14,15}$
}

Published online: 14 September 2018

(c) Springer Nature Limited 2018

Correction to: Leukemia; https://doi.org/10.1038/leu.2017. 294; published online 17 October 2017

The original version of this Article contained an error in the spelling of the author Juan Carlos Rodriguez-
Manzaneque, which was incorrectly given as $\mathrm{J}$ Carlos Rodríguez-Manzaneque. This has now been corrected in both the PDF and HTML versions of the Article.
C. Bueno

cbueno@carrerasresearch.org

$\triangle$ P. Menéndez pmenendez@carrerasresearch.org

1 Josep Carreras Leukemia Research Institute, Department of Biomedicine, School of Medicine,, University of Barcelona, Barcelona, Spain

2 Erasmus University Medical Center, Rotterdam, The Netherlands

3 Princess Maxima Center for Paediatric Oncology, Utrecht, The Netherlands

4 GENYO, Centre for Genomics and Oncological Research: Pfizer/ University of Granada/Andalusian Regional Government, Granada, Spain

5 Oncohematología, Hospital Universitario Niño Jesús, Madrid, Spain

6 Centro Ricerca Tettamanti, University of Milano-Bicocca, Ospedale San Gerardo Monza, Italy
7 Interfant Trial Data Center, University of Milano-Bicocca, Monza, Italy

8 Department of Pediatric Hemato-Oncology, Hannover Medical School, Hannover, Germany

9 Pathology Service, Hospital del Mar, Barcelona, Spain

10 Pediatric Hematology, A. Trousseau Hospital, Paris, France

11 Developmental Tumor Biology Laboratory, Hospital Sant Joan de Deu, Barcelona, Spain

12 Department of Pediatric Hematology and Oncology, Ospedale Bambino Gesù, Rome, University of Pavia, Pavia, Italy

13 Biomed Division, LEITAT Technological Centre, Barcelona, Spain

14 Centro de Investigacion Biomedica en Red-Oncología (CIBERONC), Barcelona, Spain

15 Instituciò Catalana de Recerca i Estudis Avançats (ICREA), Barcelona, Spain 